\title{
A High-Efficient Line-Based Architecture for 2-D Lifting-Based DWT Using 9/7 Wavelet Filters
}

\author{
Tze-Yun Sung ${ }^{1}$ Chun-Wang Yu ${ }^{1} \quad$ Yaw-Shih Shieh $^{1}$ Hsi-Chin Hsin ${ }^{2}$ \\ ${ }^{1}$ Department of Microelectronics Engineering, Chung Hua University, Hsinchu, Taiwan 300-12 \\ ${ }^{2}$ Department of Computer Science and Information Engineering, Formosa University, Hu-Wei, Taiwan 632-08
}

\begin{abstract}
In this paper, a high-efficient lined-based architecture for the 9/7 discrete wavelet transform (DWT) based on lifting scheme is proposed. The proposed parallel and pipelined architecture consists of a horizontal filter (HF) and a vertical filter (VF). The critical path of the proposed architecture is reduced. Filter coefficients of the biorthogonal 9/7 wavelet low-pass filter are quantized before implementation in the high-speed computation hardware. In the proposed architecture, all multiplications are performed using less shifts and additions.

The proposed architecture is $100 \%$ hardware utilization and ultra low-power. The architecture has regular structure, simple control flow, high throughput and high scalability. Thus, it is very suitable for newgeneration image compression systems, such as JPEG2000 .
\end{abstract}

Keywords: 9/7 discrete wavelet transform (DWT), horizontal filter (HF), vertical filter (VF), lined-based architecture, lifting scheme, JPEG-2000.

\section{Introduction}

In the field of digital image processing, the JPEG2000 standard uses the scalar wavelet transform for image compression [1]; hence, the two-dimensional (2-D) discrete wavelet transform (DWT) has recently been used as a powerful tool for image coding/decoding systems. Two-dimensional DWT demands massive computations, hence, it requires a parallel and pipelined architecture to perform real-time or on-line video and image coding and decoding, and to implement high-efficiency application-specific integrated circuits (ASIC) or field programmable gate array (FPGA). At the kernel of the compression stage of the system is the DWT.

Swelden proposed using the biorthogonal 9/7 wavelet based on lifting scheme for lossy compression [2]. The symmetry of the biorthogonal 9/7 filters and the fact that they are almost orthogonal [2] make them good candidates for image compression application. The coefficients of the filter are quantized before hardware implementation; hence, the multiplier can be replaced by limited quantity of shift registers and adders. Thus, the system hardware is saved, and the system throughput is improved significantly.

In this paper, we proposed a high-efficient architecture for the even and odd parts of 1-D DWT based on lifting scheme. The advantages of the proposed architectures are $100 \%$ hardware- utilization, multiplierless, regular structure, simple control flow and high scalability.

The remainder of the paper is organized as follows. Section 2 presents the lifting-based 2-D discrete wavelet transform algorithm, and derives new mathematical formulas. In Section 3, the high-efficient architecture for the lifting-based 2-D DWT is proposed. Finally, comparison of performance between the proposed architectures and previous works is made with conclusions given in Section 4.

\section{The Lifting-Based 2-D DIWT Algorithm}

Usually the Lifting-based DWT requires less computation compared to the convolution-based approach. However, the savings depend on the length of the filters. During the lifting implementation, noextra memory buffer is required because of the inplace computation feature of lifting. This is particularly suitable for the hardware implementation with limited available on-chip memory. Many papers proposed the algorithms and architectures of DWT [3], [4], [5], [6], [7], [8], [9], but they require massive computation. In 1996, Sweldens proposed a new lifting-based DWT architecture, which requires half of hardware compared to the conventional approaches [2]. The discrete wavelet transform factoring into lifting scheme is represented as [10]:

$\widetilde{P}=\left[\begin{array}{cc}1 & \alpha\left(1+z^{-1}\right) \\ 0 & 1\end{array}\right] \begin{array}{cc}1 & 0 \\ \beta(1+z) & 1\end{array}\left[\begin{array}{cc}1 & \gamma\left(1+z^{-1}\right) \\ 0 & 1\end{array}\right]\left[\begin{array}{cc|cc}1 & 0 & \zeta & 0 \\ \delta(1+z) & 1 & 0 & 1 / \zeta\end{array}\right]$

where $\alpha, \beta, \gamma$ and $\delta$ are the coefficients of lifting scheme, and $\zeta$ and $1 / \zeta$ are scale normalization factors.

The architecture based on lifting scheme consists of splitting module, two lifting module and 
scaling module. The architecture of 9/7 1-D DWT based on lifting scheme is shown in Figure 1.

\subsection{The 9/7 2-D DWT Algorithm}

According to the architecture of 9/7 1-D DWT based on lifting scheme, the architecture of modified 9/7 2-D DWT based on lifting scheme can be derived and shown in Figure 2. The equations of the 2-D DWT based on lifting scheme is represented as The horizontal filter (HF) is represented as:

$$
\begin{aligned}
& H^{1}(i, j)=x(i, 2 j+1)+\alpha(x(i, 2 j)+x(i, 2 j+2)) \\
& L^{1}(i, j)=x(i, 2 j)+\beta\left(H^{1}(i, j)+H^{1}(i, j-1)\right) \\
& H^{2}(i, j)=H^{1}(i, j)+\gamma\left(L^{1}(i, j)+L^{1}(i, j+1)\right. \\
& L^{2}(i, j)=L^{1}(i, j)+\delta\left(H^{2}(i, j)+H^{2}(i, j-1)\right)
\end{aligned}
$$

The vertical filter (VF) is represented as:

$$
\text { High frequency part: }
$$

$$
\begin{aligned}
& H H^{1}(i, j)=H^{2}(2 i+1, j)+\alpha\left(H^{2}(2 i, j)+H^{2}(2 i+2, j)\right) \\
& H L^{1}(i, j)=H^{2}(2 i, j)+\beta\left(H H^{1}(i, j)+H H^{1}(i-1, j)\right) \\
& H H^{2}(i, j)=H H^{1}(i, j)+\gamma\left(H L^{1}(i, j)+H L^{1}(i+1, j)\right) \\
& H L^{2}(i, j)=H L^{1}(i, j)+\delta\left(H H^{2}(i, j)+H H^{2}(i-1, j)\right) \\
& H H(i, j)=H H^{2}(i, j) \times 1 / \zeta^{2} \\
& H L(i, j)=H L^{2}(i, j) \\
& L o w \text { frequency part: } \\
& L H^{1}(i, j)=L^{2}(2 i+1, j)+\alpha\left(L^{2}(2 i, j)+L^{2}(2 i+2, j)\right) \\
& L L^{1}(i, j)=L^{2}(2 i, j)+\beta\left(L H^{1}(i, j)+L H^{1}(i-1, j)\right) \\
& L H^{2}(i, j)=L H^{1}(i, j)+\gamma\left(L L^{1}(i, j)+L L^{1}(i+1, j)\right) \\
& L L^{2}(i, j)=L L^{1}(i, j)+\delta\left(L H^{2}(i, j)+L H^{2}(i-1, j)\right) \\
& L H(i, j)=L H^{2}(i, j) \\
& L L(i, j)=L L^{2}(i, j) \times \zeta^{2}
\end{aligned}
$$

\subsection{The modified 9/7 2-D DWT Algorithm}

According to eq. (1), the transform matrix of the 9/7 DWT based on lifting scheme is modified as

$$
\begin{aligned}
\tilde{P}_{1}(z) & =\left[\begin{array}{cc}
1 & \alpha\left(1+z^{-1}\right) \\
0 & 1
\end{array}\right]\left[\begin{array}{cc}
1 & 0 \\
\beta(1+z) & 1
\end{array}\right]\left[\begin{array}{cc}
1 & \gamma\left(1+z^{-1}\right) \\
0 & 1
\end{array}\right]\left[\begin{array}{cc}
1 & 0 \\
\delta(1+z) & 1
\end{array}\right]\left[\begin{array}{cc}
\zeta & 0 \\
0 & 1 / \zeta
\end{array}\right] \\
& \left.\left.=\left[\begin{array}{cc}
1 / \alpha & \left(1+z^{-1}\right) \\
0 & 1 / \alpha
\end{array}\right]\left[\begin{array}{cc}
1 / \beta & 0 \\
(1+z) & 1 / \beta
\end{array}\right] \begin{array}{cc}
1 / \gamma & \left(1+z^{-1}\right) \\
0 & 1 / \gamma
\end{array}\right]\left[\begin{array}{cc}
1 / \delta & 0 \\
1+z) & 1 / \delta
\end{array}\right] \begin{array}{cc}
\alpha \beta \gamma \delta \zeta & 0 \\
0 & \alpha \beta \gamma \delta \zeta
\end{array}\right] \\
& =\left[\begin{array}{cc}
1 & 1+z^{-1} \\
0 & 1 / \alpha
\end{array}\right]\left[\begin{array}{cc}
1 / \alpha \beta & 0 \\
1+z & 1
\end{array}\right]\left[\begin{array}{cc}
1 & 1+z^{-1} \\
0 & 1 / \beta \gamma \\
1 / \gamma \delta & 0 \\
1+z & 1
\end{array}\right]\left[\begin{array}{cc}
\alpha \beta \gamma \delta \zeta & 0 \\
0 & \alpha \beta \gamma / \zeta
\end{array}\right] \\
& =\left[\begin{array}{cc}
1 & 1+z^{-1} \\
0 & A
\end{array}\right]\left[\begin{array}{cc}
B & 0 \\
1+z & 1
\end{array}\right]\left[\begin{array}{cc}
1 & 1+z^{-1} \\
0 & C
\end{array}\right]\left[\begin{array}{cc}
D & 0 \\
1+z & 1
\end{array}\right]\left[\begin{array}{cc}
K_{0} & 0 \\
0 & K_{1}
\end{array}\right]
\end{aligned}
$$

where

$$
\begin{aligned}
& A=1 / \alpha, B=1 / \alpha \beta, C=1 / \beta \gamma, D=1 / \gamma \delta, K_{0}=\alpha \beta \gamma \delta \zeta \text { and } \\
& K_{1}=\alpha \beta \gamma / \zeta .
\end{aligned}
$$

The modified horizontal filter (HF) is represented as:

$$
\begin{aligned}
& H^{1}(i, j)=A \times x(i, 2 j+1)+x(i, 2 j)+x(i, 2 j+2) \\
& L^{1}(i, j)=B \times x(i, 2 j)+H^{1}(i, j)+H^{1}(i, j-1) \\
& H^{2}(i, j)=C \times H^{1}(i, j)+L^{1}(i, j)+L^{1}(i, j+1)
\end{aligned}
$$

$L^{2}(i, j)=D \times L^{1}(i, j)+H^{2}(i, j)+H^{2}(i, j-1)$

The modified vertical filter (VF) is represented as:

High frequency part:

$H H^{1}(i, j)=A \times H^{2}(2 i+1, j)+H^{2}(2 i, j)+H^{2}(2 i+2, j)$

$H L^{1}(i, j)=B \times H^{2}(2 i, j)+H H^{1}(i, j)+H H^{1}(i-1, j)$

$H H^{2}(i, j)=C \times H H^{1}(i, j)+H L^{1}(i, j)+H L^{1}(i+1, j)$

$H L^{2}(i, j)=D \times H L^{1}(i, j)+H H^{2}(i, j)+H H^{2}(i-1, j)$

Low frequency part:

$L H^{1}(i, j)=A \times L^{2}(2 i+1, j)+L^{2}(2 i, j)+L^{2}(2 i+2, j)$

$L L^{1}(i, j)=B \times L^{2}(2 i, j)+L H^{1}(i, j)+L H^{1}(i-1, j)$

$L H^{2}(i, j)=C \times L H^{1}(i, j)+L L^{1}(i, j)+L L^{1}(i+1, j)$

$L L^{2}(i, j)=D \times L L^{1}(i, j)+L H^{2}(i, j)+L H^{2}(i-1, j)$

Finally, four subbands of $H H, H L, L H$ and $L L$ are performed by $H H^{2}, H L^{2}, L H^{2}$ and $L L^{2}$. The equations of four subbands are represented as follows: $H H(i, j)=H H^{2}(i, j) \times K_{0}^{2}=H H^{2}(i, j) \times \alpha^{2} \beta^{2} \gamma^{2} \delta^{2} \zeta^{2}$ $H L(i, j)=H L^{2}(i, j) \times K_{1} K_{0}=H L^{2}(i, j) \times \alpha^{2} \beta^{2} \gamma^{2} \delta$ $L H(i, j)=L H^{2}(i, j) \times K_{1} K_{0}=L H^{2}(i, j) \times \alpha^{2} \beta^{2} \gamma^{2} \delta$ $L L(i, j)=L L^{2}(i, j) \times K_{1}^{2}=L L^{2}(i, j) \times \alpha^{2} \beta^{2} \gamma^{2} / \zeta^{2}$

According to the equations of modified horizontal filter (HF), the architecture for modified horizontal filter (HF) is proposed and shown in Figure 3. The proposed architecture for modified horizontal filter (HF) consists of input-delay unit, middle-delay unit, back-delay unit, five multiplexers and two processing elements (PEs). The $\mathrm{PE}(\mathrm{A} / \mathrm{B})$ performs $\mathrm{O} 1$ and $\mathrm{PE}(\mathrm{C} / \mathrm{D})$ performs O2. Similarly, the proposed architecture for modified vertical filter (VF) is shown in Figure 4. The proposed architecture for modified vertical filter (VF) consists of two delay units (Ds), seven long-delay (8 Ds) units, eight multiplexers and two processing elements $(\mathrm{PE}(\mathrm{A} / \mathrm{B})$ and $\mathrm{PE}(\mathrm{C} / \mathrm{D}))$. The architecture of scaling normalization (SN) is shown in Figure 5. The new architecture of $\mathrm{PE}$ is shown in Figure 6. The proposed PE architecture reduces the critical path [11] [12]. The 2-D DWT system is shown in Figure 7.

\section{The High-Efficient Architecture for Lifting-Based 2-D DWT}

In $8 \times 82$-D DWT, it requires 106 clocks to perform 2D DWT. Clock cycles 2 to 66 perform O1, clock cycles 7 to 70 perform $\mathrm{O} 2$, clock cycles 24 to 87 perform O3, and clock cycles42 to 106 perform $\mathrm{O} 4$. Every PE requires $N \times N$ clocks to perform the output.

Filter coefficients of the biorthogonal 9/7 wavelet low-pass filter are quantized before implementation in the high-speed computation hardware. In the proposed architecture, all multiplications are performed using shifts and additions after approximating the coefficients as a booth binary recoded format. The 
multiplier is replaced by a carry-save-adder (CSA) and three hardwire shifters in processing element (PE) [13].

\section{Conclusions and Discussions}

Filter coefficients are quantized before implementation using the biorthogonal $9 / 7$ wavelet. The hardware is cost-effective and the system is high-speed. The architecture reduces power dissipation by $m$ compared with conventional architectures in $m$-bit operand (lowpower utilization).

In this paper, the high-efficient and low-power architecture for 2-D DWT have been proposed. The architecture performs compression in $4 \cdot\left(1-2^{-2 j}\right) \cdot N^{2} / 3 \cdot T_{a}$ computation time, where the time unit ( $T_{a}$ is time of addition operation). The critical path is $2 T_{a}$ and the output latency time is $4 N \cdot T_{a}+8$. The buffer size is $N^{2} / 4+7 N+11$. The control complexity is simple. The comparison between previous works and this work is shown in Table 1.

The proposed architecture has been verified by Verilog-HDL and implemented on FPGA. The advantages of the proposed architecture are 100\% hardware utilization and ultra low-power. The architecture has regular structure, simple control flow, high throughput and high scalability. Thus, it is very suitable for new-generation image compression systems, such as JPEG-2000.

\section{References}

[1] ITU-T Recommendation T.800. JPEG2000 image coding system- Part 1, ITU Std., July 2002. http://www.itu.int/ITU-T/.

[2] W. Sweldens, "The lifting scheme: A customdesign construction of biorthogonal wavelet," Applied and Computational Harmonic Analysis, vol. 3, pp.186-200, 1996.

[3] G. Beylkin R. Coifman, and V. Rokhlin, Wavelet in Numerical Analysis in Wavelets and their
Applications. New York: Jones and Bartlett,1992.

[4] A. N. Akansu and R. A. Haddad, Multiresolution Signal Decomposition: Transform, Subbands and Wavelets. New York: Academic, 1992

[5] I. Sodagar, H.-J. Lee, P. Hatrack, and Y.-Q. Zhang, "Scalable wavelet coding for synthetic/natural hybrid images," IEEE Trans. Circuits and Systems for Video Technology, vol. 9, pp. 244254, March 1999.

[6] D. Taubman, "High performance scalable image compression with EBCOT," IEEE Trans. Image Processing, vol. 9, pp. 1158-1170, July 2000.

[7] R. Kronland-Martinet, J. Morlet, and A. Grossman, "Analysis of sound patterns through wavelet transform," Int. J. Pattern Recognit. Artif. Intell., vol. 1, no. 2, pp. 273-302, 1987.

[8] M. A. Stoksik, R. G. Lane, D. T. Nguyen, "Accurate synthesis of fractional Brownian motion using wavelets," Electronic Letters, vol. 30, no. 5, pp. 384-284, March 1994.

[9] K. Parhi and T. Nishitani, "VLSI architectures for discrete wavelet transforms," IEEE Trans. VLSI Systems, vol. 1, no. 2, pp. 191-202, 1993.

[10] I. Daubechies and W.Sweldens, "Factoring wavelet transforms into lifting schemes," J.Fourier Anal. Appl., vol.4, pp.247-269, 1998.

[11] K. Andra, C. Chakrabarti, T. Acharya, "A VLSI architecture for lifting-based forward and inverse wavelet transform," IEEE Transactions on Signal Processing, vol. 50, no. 4, pp. 966-977, 2002.

[12] C. Xiong, S. Zheng, J. Tian, J. Liu, "The improved lifting scheme and novel reconfigurable VLSI architecture for the 5/3 and 9/7 wavelet filters," 2004 IEEE International Conference on Communications, Circuits and Systems, vol. 2, pp. 728-732, 2004.

[13] T. Y. Sung, Y. S. Shieh, "A High-Speed / Ultra Low-Power Architecture for 2-D Discrete Wavelet Transform," 2005 IEEE International Conference on Systems and Signals (ICSS-2005), I-Shou University, Kaohsiung, Taiwan, April 2829,pp.326-331,2005.

Table 1 The comparison between previous works and this work

\begin{tabular}{|c|c|c|}
\hline Architecture & Andra [11] & This work \\
\hline Buffer size & $N^{2}$ & $N^{2} / 4+7 N+11$ \\
\hline Multipliers & 6 & --- \\
\hline Adders & 8 & 13 \\
\hline Output latency & $N^{2} \cdot T_{m}$ & $4 N \cdot T_{a}+8$ \\
\hline Critical Path & $T_{m}+2 \cdot T_{a}$ & $2 \cdot T_{a}$ \\
\hline Computation time & $4 \cdot\left(1-2^{-2 j}\right) \cdot N^{2} / 3 \cdot T_{m}$ & $4 \cdot\left(1-2^{-2 j}\right) \cdot N^{2} / 3 \cdot T_{a}$ \\
\hline Control complexity & medium & simple \\
\hline Power consumption & High & Low \\
\hline Chip area & Large & High \\
\hline Throughput & Low & \\
\hline
\end{tabular}




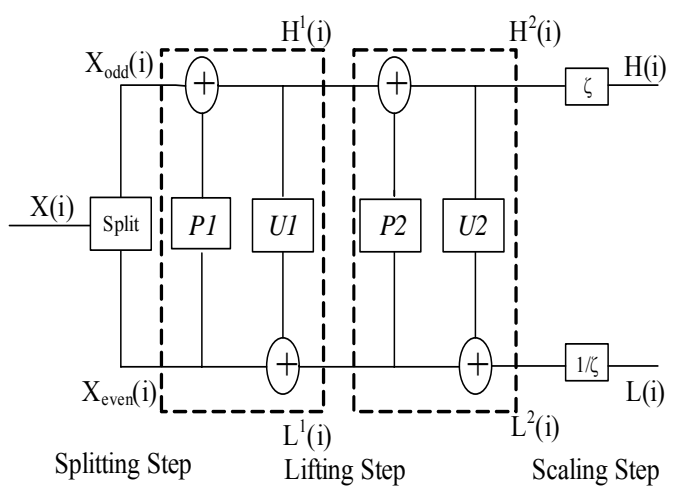

Fig. 1. The architecture of 9/7 1-D DWT based on lifting scheme $\left(P l=\alpha(l+z), \quad U 1=\beta\left(l+z^{-1}\right)\right.$, $P 2=\gamma(1+z)$ and $\left.U 2=\delta\left(1+z^{-1}\right)\right)$

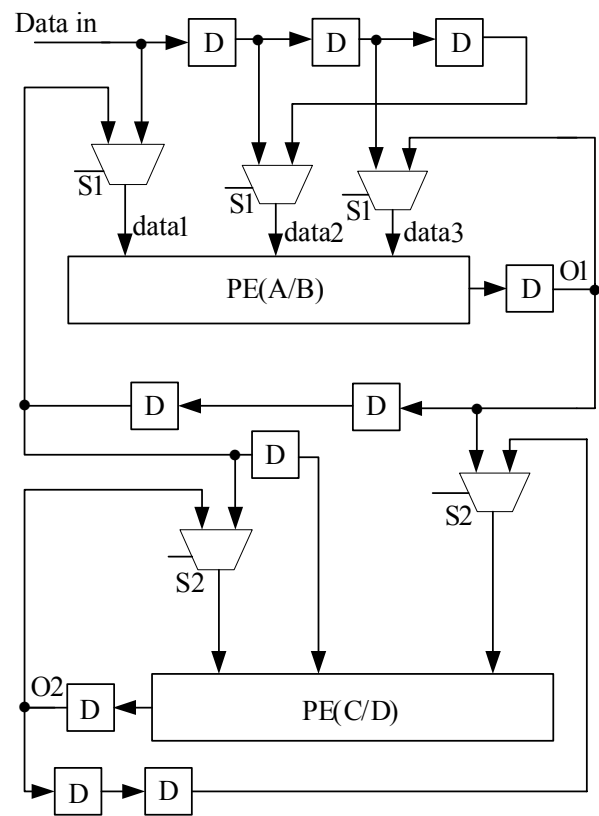

Fig.3. The proposed architecture for horizontal filter (D: delay unit, S1,S2:select inputs for multiplexers)

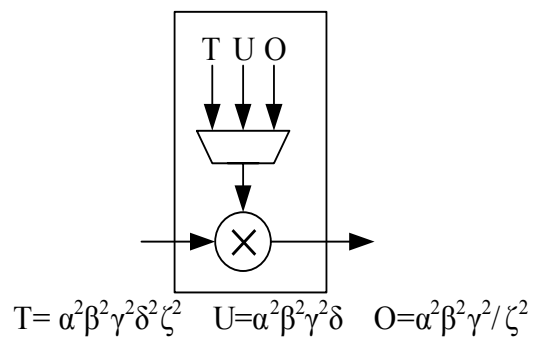

Fig. 5. The architecture for scaling normalization

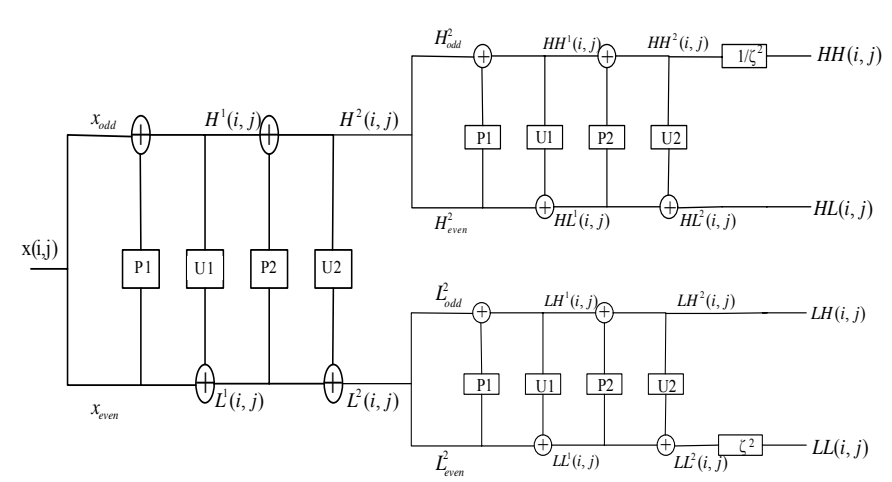

Fig. 2. The architecture of modified $9 / 7$ lifting-based DWT

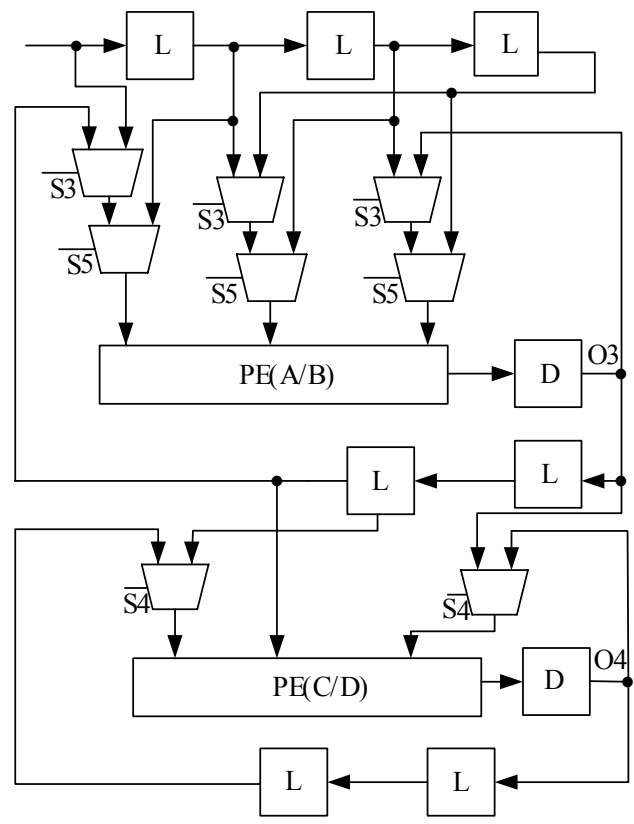

Fig.4. The architecture for vertical filter (D:delay unit, L:long-delayunit, S3,S4,S5:select inputs for multiplexers)

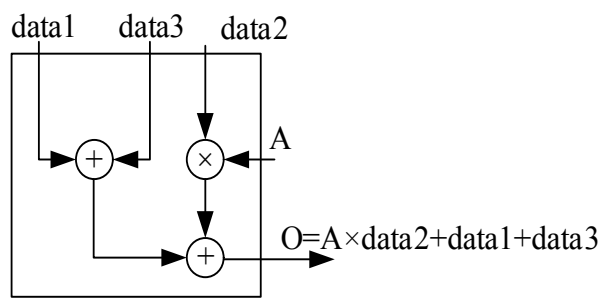

Fig. 6. The new architecture of $\mathrm{PE}$

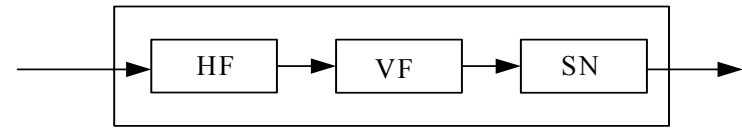

Fig.7. The 2-D DWT system 